\title{
Passive permeability and outward active transport of fluorescein across the blood-retinal barrier in early ARM
}

\author{
Birgitte Moldow, Michael Larsen, Birgit Sander, Henrik Lund-Andersen
}

\begin{abstract}
Aim-To study the passive and active transport of fluorescein across the bloodretina barrier in early age related maculopathy (ARM) (soft drusen > $>3 \mu \mathrm{m}$, hyperpigmentation and/or hypopigmentation in patients above 50 years of age). Methods-15 patients and 10 healthy subjects were included. Morphological changes were graded from 30 degrees fundus photographs using a simplified version of the epidemiological ARM study group classification system. Differential vitreous spectrofluorophotometry was used to assess the transport properties of the blood-retina barrier (that is, passive permeability and unidirectional permeability caused by outward active transport from the vitreous to the blood).

Results-The passive permeability of the patient group was not significantly different from that of the control group. Four patients with passive permeability more than $3 \mathrm{SD}$ above the mean of the control group (mean $1.8(\mathrm{SD} 0.7) \mathrm{nm} / \mathrm{s}$, range 1.0 $3.0 \mathrm{~nm} / \mathrm{s}$, data normally distributed) all had centrally located drusen $>500 \mu \mathrm{m}$ and superjacent pigment clumps of 63-500 $\mu \mathrm{m}$ in diameter. There was no significant difference between the unidirectional permeabilities for the patient group and for the control group (mean $47.4(29.3) \mathrm{nm} / \mathrm{s}$, range $12.7-91.1 \mathrm{~nm} / \mathrm{s})$.

Conclusion-There was no significant difference in the passive permeability and in the unidirectional permeability of fluorescein. However, the study may indicate that the combination of very large drusen and superjacent pigment clumps in ARM may be associated with a deterioration of the blood-retina barrier.

(Br F Ophthalmol 2001;85:592-597)
\end{abstract}

Department of Ophthalmology, Herlev Hospital, University of Copenhagen, Denmark B Moldow

M Larsen

B Sander

$\mathrm{H}$ Lund-Andersen

Correspondence to:

Birgitte Moldow, $\mathrm{PhD}$,

Soløsevej 39, DK- 2820

Gentofte, Denmark

b.moldow@ofir.dk

Accepted for publication 27 November 2000
Early age related maculopathy (ARM) - that is, soft drusen $>63 \mu \mathrm{m}$, hyperpigmentation and/or hypopigmentation in patients above 50 years of age with soft drusen, ${ }^{1}$ is known to be associated with changes in the pigment epithelium and deposits localised between the pigment epithelium and Bruch's membrane, ${ }^{23}$ Histopathological changes in the pigment cell layer are typically flattening and abnormal proliferation..$^{2-4}$ Drusen deposits appear to have derived from the pigment epithelium cells, ${ }^{56}$ and it has been suggested that soft drusen may reflect a diffuse dysfunction of the pigment epithelium. ${ }^{78}$
Recent research has focused on the normal age related deposits and on the importance of the drusen deposits for the normal exchange of nutrients, electrolytes, and fluids between the choroid and the pigment epithelium. It has been argued that these deposits, which contain lipids, ${ }^{59}$ may function as a hydrophobic barrier inhibiting the passage of hydrophilic matter and fluid. By making more difficult the passage of ions that are actively pumped across the pigment epithelium and of fluids in the direction from the retina to the blood, this hydrophobic barrier is assumed to predispose to retention of fluid and thereby development of pigment epithelium detachment. ${ }^{10} 11$

Vitreous fluorometry using fluorescein as tracer is a method for quantification of the passive permeability of the blood-retina barrier and the active outward transport of fluorescein. ${ }^{12}$ Fluorescein is assumed to pass the blood-retina barrier by passive diffusion and by outward active transport from vitreous to blood.

This study investigates whether early ARM is related to changes in the active transport of fluorescein. It is probable that a dysfunction of the pigment epithelium is associated with an effect on the active transport mechanisms which may also impact the active transport of fluorescein. Furthermore, the study considers whether early ARM is associated with changes in the passive transport. Although the pigment epithelium is seen as the outer barrier ${ }^{13}$ it is unclear whether the deposits would be able to affect the passive permeability across the blood-retina barrier.

The study may contribute to a better pathogenic understanding of the development of early stages of age related maculopathy.

\section{Materials and methods}

According to the classification system of the epidemiological ARM study group, the diagnosis of early ARM has been defined as the presence of soft drusen $>63 \mu \mathrm{m}$, with any hyperpigmentation and/or hypopigmentation in patients over 50 years of age. Hard drusen $<125 \mu \mathrm{m}$ is not included in the definition. ${ }^{1}$

A total of 17 patients with early ARM and absence of glaucoma, cataract, and other eye diseases in at least one eye gave their informed consent to participate in the study. The included patients were referred to the ophthalmology department, Herlev University Hospital. Two patients were found by screening volunteers for the control group. Two patients were excluded a priori, because of arterial hypertension (BP >160/90) and one because of diabetes mellitus. The remaining group of 15 
patients consisted of nine men and six women, all without other known systemic illness and without allergy or intolerance for fluorescein.

Ten subjects, five men and five women, volunteered for the control group and gave their informed consent to participate in the study. All were without ARM and with absence of glaucoma, cataract, and other eye diseases in at least one eye and complied with the same other exclusion criteria as the patient group.

All participants were white people. The age distribution for the group of patients and controls was comparable (Tables 1 and 2). The median age was 67 years (range 55-72) in the patient group and 66 years (range 52-69) in the control group. The age distribution for the group of patients and controls (Tables 1 and 2) was not statistically different (Mann-Whitney, non-parametric test for unpaired data, level of significance $<0.05$ ).

For each participant only one eye was included. Late ARM as defined by the ARM study group, ${ }^{1}$ was found in the fellow eyes of five people, exudative changes in four cases, and geographic atrophy in one case. In the control group, four eyes in four patients were excluded owing to pseudophakia following a cataract operation, significant cataract, amblyopia, and a focal pigment epitheliopathy. For the remaining subjects the eye to be included was chosen on the basis of a fluorometric study of the vitreous diffusion coefficient of fluorescein. From experience, incipient degenerative changes in the vitreous are known to be associated with a high variation of the vitreous diffusion coefficient and with high apparent vitreous diffusion coefficients. ${ }^{14}$ To optimise the determination of the blood-retina barrier transport parameters, the eye with the lowest variation of the diffusion coefficient was selected for each subject.

The study followed the tenets of the Declaration of Helsinki, and the protocol was approved by the local ethics committee and the Danish National Board of Health.

All participants underwent an examination including determination of arterial blood pressure, determination of visual acuity (ETDRS standard logarithmic charts), slit lamp examination, biomicroscopy, ophthalmoscopy, colour fundus photography (Canon camera CF60UV, Ectachrome 64 film, stereo at 30, 40, and 60 degrees), fluorescein angiography (HP5 film), and fluorometric examination.

GRADING ARM

The grading of early ARM was performed by an experienced investigator (ML) who was unaware of the fluorometric results. A simplified version of the international ARM study group grading system was used to assess 30 degree stereo photographs of the macula. ${ }^{1} \mathrm{~A}$ transparency with standard circles with an inner and outer diameter of 1000 and $6000 \mu \mathrm{m}$ was centred over the fovea. The size of the largest soft drusen central to the outer ring was estimated by use of standard circles with diameters of $63,125,175,250$, and $500 \mu \mathrm{m}$ and graded as $<63 \mu \mathrm{m} ; \geqslant 63 \mu \mathrm{m}$ and $<125 \mu \mathrm{m}$; $\geqslant 125 \mu \mathrm{m}$ and $<175 \mu \mathrm{m} ; \geqslant 175 \mu \mathrm{m}$ and $<250$ $\mu \mathrm{m}, \geqslant 250 \mu \mathrm{m}$ and $<500 \mu \mathrm{m}$; and $\geqslant 500 \mu \mathrm{m}$. Focal hyperpigmentation was graded according to size in $<63 \mu \mathrm{m}$; $\geqslant 63 \mu \mathrm{m}$ and $<125 \mu \mathrm{m}$; $\geqslant 125 \mu \mathrm{m}$ and $<175 \mu \mathrm{m} ; \geqslant 175 \mu \mathrm{m}$ and $<250$ $\mu \mathrm{m}$; and $\geqslant 250 \mu \mathrm{m}$. The largest focal hyperpigmentation and its localisation in relation to the drusen were noted. It was recorded whether the drusen changes involved the area central to the inner ring.

FLUOROMETRIC DETERMINATION OF PASSIVE AND UNIDIRECTIONAL PERMEABILITY

The fluorometric method and its assumptions for determination of the passive diffusion and outward active transport have been discussed in detail in earlier studies. ${ }^{15-18}$ A summary of the method follows below.

The transport of fluorescein from the blood to the retina is assumed to be transported by pure passive diffusion as discussed previously. ${ }^{13}$ The transport of fluorescein from retina to blood, is assumed to include active transport, based on animal studies. ${ }^{19}{ }^{20}$ There is no evidence of other contributors to the transport of fluorescein across the blood-retina barrier to our knowledge. Several studies have refuted a significant posteriorly directed bulk flow. ${ }^{21} 22$ Any contribution to the transport of fluorescein due to an electrical potential is thought to be small. ${ }^{23}$

\section{INSTRUMENTATION}

Ocular fluorescence measurements were performed by use of a differential spectrofluorometry technique using a commercially available fluorometer (Fluorotron, Ocumetics Inc, CA, USA) equipped with a modified light source which excites light at rapidly interchanging wavelengths of 458 and $488 \mathrm{~nm} .{ }^{17}$ For the plasma fluorescence analysis, a cuvette spectrofluorometre (Perkin Elmer LS 50) was used.

\section{PROCEDURE}

The investigations commenced in the morning. An intravenous catheter was positioned in a superficial antecubital arm vein, and the pupils were dilated using topical $10.0 \%$ phenylephrine plus $1 \%$ tropicamide.

Patients received an intravenous bolus injection of sodium fluorescein $(14 \mathrm{mg} / \mathrm{kg}$ body weight). Measurements of ocular fluorescence in the optical axis of the eye were conducted before the injection, and at 30 minutes, 7, 8, 9, and 10 hours after injection (three scans per measurement at 30 minutes after injection, thereafter four scans per measurement). Sufficient dilatation of the pupil (more than $7 \mathrm{~mm}$ ) was ensured before every fluorescence measurement using supplementary eye drops. Plasma fluorescein concentrations were determined from blood samples drawn from the catheter before injection, and after approximately 5, 7, 10, 15, 30 minutes, and 1, 2, 7, 8, 9 , and 10 hours after injection as described in an earlier study. ${ }^{14}$

VITREOUS CONCENTRATION DETERMINATION

The recorded scans were aligned after fluorescence landmarks (cornea and the lens). The 
Table 1 Data for 15 patients with early ARM. The table lists age, visual acuity, drusen diameter, focal hyperpigmentation, $P^{(p a s)}$ and $P^{(u n i)}$ and any late changes in the fellow eye. $P^{(u n i)}$ for patient no 1 is unavailable

\begin{tabular}{|c|c|c|c|c|c|c|c|}
\hline $\begin{array}{l}\text { Patient } \\
\text { (No) }\end{array}$ & $\begin{array}{l}\text { Age } \\
\text { (years) }\end{array}$ & Visual acuity & $\begin{array}{l}\text { Drusen } \\
\text { diameter }(\mu m)\end{array}$ & $\begin{array}{l}\text { Focal hyperpigmentatio } n \\
\text { (diameter, } \mu m)\end{array}$ & $\begin{array}{l}P^{(p a s)} \\
(n m / s)\end{array}$ & $\begin{array}{l}P^{(u m i)} \\
(n m / s)\end{array}$ & $\begin{array}{l}\text { Other eye, late ARM } \\
\text { changes }\end{array}$ \\
\hline 1 & 62 & $20 / 25-1$ & $>500$ & $\begin{array}{l}\text { Clumps above drusen } \\
(63-175)\end{array}$ & 8.12 & - & \\
\hline 2 & 68 & $20 / 100-2$ & $>500$ & $\begin{array}{l}\text { Clumps above drusen } \\
(>250)\end{array}$ & 18.40 & 35,7 & \\
\hline 3 & 72 & $>20 / 20$ & $250-500$ & & 2.78 & 33,8 & Exudative changes \\
\hline 4 & 67 & $20 / 25$ & $250-500$ & & 1.52 & 122,1 & \\
\hline 5 & 70 & $20 / 100+1$ & $>500$ & $\begin{array}{l}\text { Clumps above drusen } \\
(175-250)\end{array}$ & 3.87 & 24,2 & \\
\hline 6 & 66 & $20 / 25-2$ & $175-250$ & $\begin{array}{l}\text { Clumps above drusen } \\
(<63)\end{array}$ & 2.25 & 25.3 & Exudative changes \\
\hline 7 & 72 & $20 / 63-1$ & $>500$ & & 1.36 & 27.4 & Exudative changes \\
\hline 8 & 67 & $>20 / 20$ & $175-250$ & & 2.12 & 239.4 & Geographic atrophy \\
\hline 9 & 55 & $20 / 80$ & $175-250$ & & 1.35 & 14,5 & \\
\hline 10 & 70 & $20 / 20$ & $63-125$ & & 0.54 & 123,1 & \\
\hline 11 & 61 & $20 / 20$ & $250-500$ & & 1.72 & 19,5 & \\
\hline 12 & 64 & $20 / 20$ & $250-500$ & & 2.50 & 14,3 & Exudative changes \\
\hline 13 & 67 & $20 / 32-1$ & $>500$ & $\begin{array}{l}\text { Clumps above drusen } \\
(125-175)\end{array}$ & 6.32 & 38,6 & \\
\hline 14 & 70 & $20 / 20-2$ & $63-125$ & & 2.38 & 73,4 & \\
\hline 15 & 71 & $>20 / 20$ & $63-125$ & & 2.18 & 57.1 & \\
\hline Average SD & 67 & & & & 3.834 .49 & $60,662,8$ & \\
\hline
\end{tabular}

analysis included correction for autofluorescence of the eye, correction for absorption in the lens based on calculations of the transmittance of the lens as suggested by Zeimer and Noth, ${ }^{24}$ and correction for absorption in extrinsic fluorophores

The lens transmittance was calculated by assuming an intrinsic ratio between the front and back lens peaks of 1.2. Ocular fluorescence data were used to calculate fluorescein concentration separately from fluorescein glucuronide as described by Larsen et al. ${ }^{17}$

MATHEMATICAL ANALYSIS OF PASSIVE

PERMEABILITY

The vitreous diffusion coefficient, $\mathrm{D}$, and the passive permeability, $\mathrm{P}^{(\mathrm{pas})}$, across the bloodretina barrier was calculated based on the plasma concentration decay curves and the ocular axial fluorescence scans recorded at 30 minutes after fluorescein injection by applying a numerical solution to a mathematical diffusion mode. ${ }^{16}$ As in an earlier study of retinitis pigmentosa, ${ }^{14} 30$ minute scans were used instead of the normally 60 minute scans. This was to minimise any influence on the preretinal gradient from any stirring in smaller areas in other parts of the vitreous caused by vitreous degeneration.

The calculations assume that the barrier between blood and vitreous consists of a homogeneous spherical shell of negligible thickness. Further, it is assumed that the concentration profiles for fluorescein within the first hour are determined by the passive permeability, the concentration curve for nonprotein bound plasma fluorescein, and the diffusion characteristics of fluorescein in the vitreous. ${ }^{16}$ Within the first hours the unidirectional flux due to outward active transport of fluorescein is assumed to be negligible. ${ }^{25}$

MATHEMATICAL ANALYSIS OF ACTIVE TRANSPORT The unidirectional permeability of fluorescein in the direction from retina to the blood, $\mathrm{P}^{\text {(uni) }}$, was determined from vitreous scans recorded at $7,8,9$, and 10 hours after fluorescein injection (four scans per hour). During this time the fluorescein concentration decreases towards retina and the net transport of fluorescein is from the vitreous to the blood.

$\mathrm{P}^{(\text {uni) }}$ is assumed to be independent of the vitreous concentrations in the late measurements. Non-saturated active transport has been assumed in other studies within this area. ${ }^{125}$

At the time, $t$, the flux of fluorescein across the blood-retina barrier in the direction from the vitreous to the blood is the sum of passive flux, $\mathrm{J}^{\text {(pas) }}$ and the unidirectional flux $\mathrm{J}^{\text {(uni) }}$. (Unidirectional flux "frequently denotes the special passive transport process where the concentration in the one phase is kept at zero". In this paper the term is used for the one way flux which is caused by the existence of special transport molecules in the membrane. ${ }^{26}$ ) Fick's law of diffusion was used to determine the outward flux across the barrier based on the vitreous concentration gradient at the retina surface and the diffusion coefficient of fluorescein in the vitreous. ${ }^{12}$ In the spherical eye with radius $0 \leqslant r \leqslant a$, where $(\partial \mathrm{C} / \partial \mathrm{r})_{\mathrm{a}}$ denotes the preretinal gradient in the distance $r=a$, the outward flux at the time $t$ can be expressed as:

$$
-\mathrm{D}(\partial \mathrm{C} / \partial \mathrm{r})_{\mathrm{a}}=\mathrm{J}^{(\text {pas })}+\mathrm{J}^{(\mathrm{uni})}
$$

The passive and unidirectional flux can be expressed as

$$
\begin{aligned}
& \mathrm{J}^{\text {(pas) }}=\mathrm{P}^{(\text {pas })}\left(C(\mathrm{a}, \mathrm{t})-\mathrm{C}^{0}(\mathrm{t})\right) \\
& \mathrm{J}^{\text {(uni) }}=\mathrm{P}^{(\text {uni) }} \mathrm{C}(\mathrm{a}, \mathrm{t})
\end{aligned}
$$

where $C(a, t)$ and $C^{0}(t)$ are the concentration at the surface of the retina and the fluorescein plasma concentration at the time $t$ respectively. $\mathrm{P}^{\text {(uni) }}$ can be derived from these equations:

$$
\begin{aligned}
\mathrm{P}^{(\mathrm{uni})}= & -\mathrm{D}(\partial \mathrm{C} / \partial \mathrm{r})_{\mathrm{a}} / \mathrm{C}(\mathrm{a}, \mathrm{t})+\mathrm{P}^{(\mathrm{pas})} \quad \mathrm{C}^{0}(\mathrm{t}) / \\
& \mathrm{C}(\mathrm{a}, \mathrm{t})-\mathrm{P}^{(\mathrm{pas})}
\end{aligned}
$$

The preretinal gradient, in the direction from the vitreous to the retina, was estimated by linear regression to vitreous concentration data at a distance of $1 \mathrm{~mm}$ to $5 \mathrm{~mm}$ from the surface of the retina. The concentration at the 
Table 2 The table lists age, $P^{(\text {pas })}$ and $P^{(\text {uni })}$ for the control group comprising 10 healthy subjects. $P^{(u n i)}$ for patient no 3 is unavailable

\begin{tabular}{llll}
\hline Subject No & Age (years) & $P^{(\text {pas })}(\mathrm{nm} / \mathrm{s})$ & $P^{\text {(uni) }}(\mathrm{nm} / \mathrm{s})$ \\
\hline 1 & 60 & 2.96 & 15.8 \\
2 & 53 & 1.27 & 66.9 \\
3 & 69 & 2.07 & 61.9 \\
4 & 66 & 1.20 & 28.3 \\
5 & 58 & 2.40 & 91.1 \\
6 & 66 & 2.55 & 12.7 \\
7 & 52 & 1.49 & 25.6 \\
8 & 69 & 1.00 & 41.0 \\
9 & 67 & 2.29 & 83.5 \\
10 & 67 & 1.12 & 47.429 .3 \\
Average SD & 66 & 1.840 .70 & \\
\hline
\end{tabular}

surface of the retina was estimated by linear extrapolation of the regression line.

$\mathrm{P}^{\text {(uni) }}$ was calculated as an average of the values determined at $7,8,9$, and 10 hours.

STATISTICAL ANALYSES

The number of patients and controls required for the study was determined assuming an SD as found in our study of healthy subjects ${ }^{16}$ (13 $\mathrm{nm} / \mathrm{s}$ for $\mathrm{P}^{\text {(uni) }}$, and $0.48 \mathrm{~nm} / \mathrm{s}$ for $\left.\mathrm{P}^{(\mathrm{pas})}\right)$, a specified mean difference $\left(15 \mathrm{~nm} / \mathrm{s}\right.$ for $\mathrm{P}^{(\text {uni })}$, and 0.5 $\mathrm{nm} / \mathrm{s}$ for $\left.\mathrm{P}^{(\mathrm{pas})}\right)$, a type 1 error of $5 \%$ and type 2 error of $20 \%{ }^{27}$

The Mann-Whitney non-parametric test for unpaired data was used to compare the fluorometric data and age distribution for the patient and control group.

\section{Results}

Permeability data, visual acuity, size of drusen, size and localisation of focal hyperpigmentation, and any presence of late ARM in the fellow eye of the patient group is reproduced in Table 1. Drusen changes involved the region central to the inner ring except for two patients (nos 8 and 15). Permeability data for the control group are reproduced in Table 2 .

For $\mathrm{P}^{(\mathrm{pas})}$ there was no significant difference when comparing the patient and control groups (Tables 1 and 2). The data followed the normal distribution as assessed with the one sample Kolmogorov-Smirnov test ${ }^{27}$ (for the

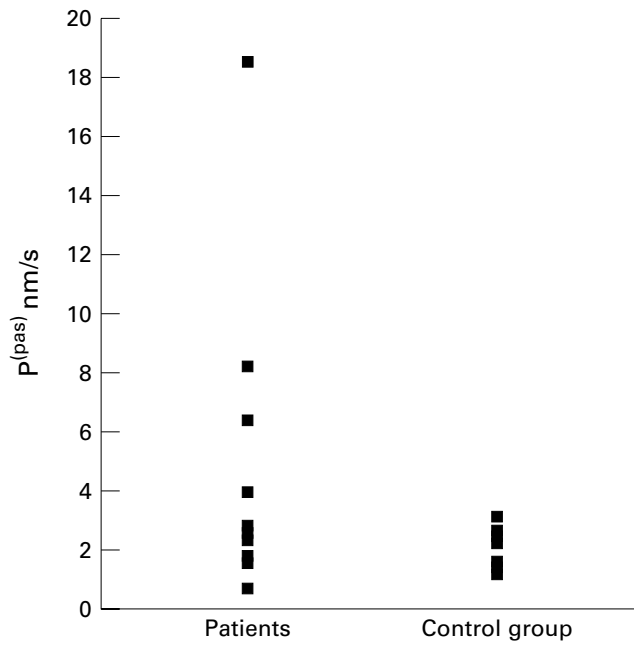

Figure 1 Passive permeabilities, $P^{(\text {pas })}$, for 15 patients with early $A R M$ and 10 controls. Four patients had central drusen $>500 \mu \mathrm{m}$ and pigment clumps 63-500 $\mathrm{um}$ in diameter intraretinally above the drusen and $P^{(p a s)}$ above the mean $+3 S D$ of the control group.

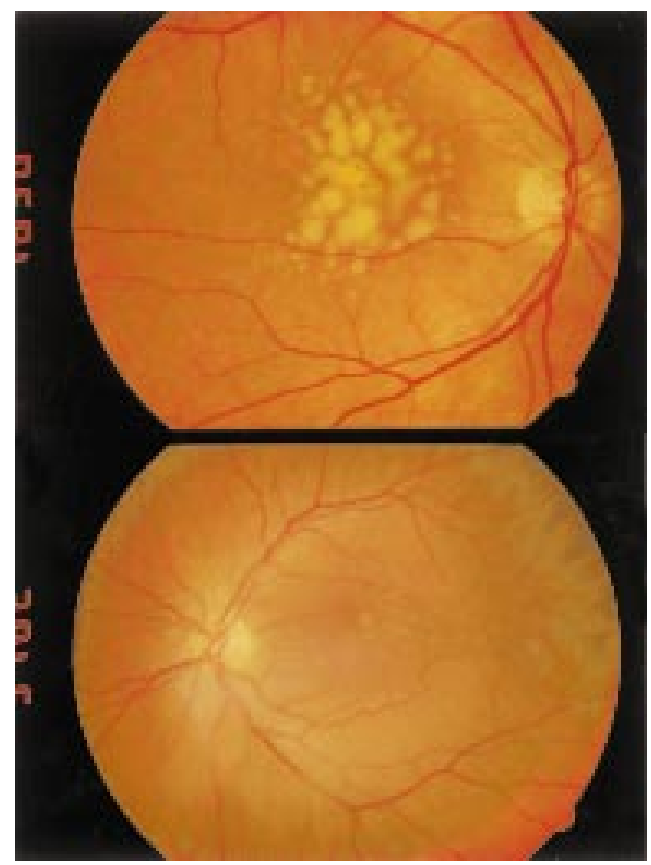

Figure 2 Fundus photo (40 degrees) from right eye of patient no 1 showing early age related maculopathy with large $(>500 \mu \mathrm{m})$ central drusen and pigment clumps intraretinally above the drusen (top). Fundus photograph (60 degrees) from the left eye of patient no 4 with moderately sized soft drusen $(250-500 \mu \mathrm{m})$ and without visible intraretinal pigment clumps (bottom).

patients after logarithmic transformation). The $\mathrm{P}^{\text {(pas) }}$ values for four patients (patients $1,2,5$, and 13) were larger or equal to the mean of the control group $+3 \mathrm{SD}$ (with one decimal) (Table 2, Fig 1). Only these four patients had drusen $>500 \mu \mathrm{m}$ and pigment clumps which were $63-500 \mu \mathrm{m}$ in diameter localised intraretinally above the drusen (Table 1 and Fig 2).

For $\mathrm{P}^{\text {(uni) }}$ there was no significant difference when comparing the patient and control groups (Tables 1 and 2 ). $P^{(\text {uni) }}$ results are unavailable for one patient and one control because of technical problems with the fluorometer in late fluorometric measurements.

There was no significant difference when comparing the diffusion coefficients of the patient and control groups. The average diffusion coefficient of the patient group was $7.4 \times$ $10^{-6}\left(5.4 \times 10^{-6}\right) \mathrm{cm}^{2} / \mathrm{s}$ (mean (SD); range 3.5$\left.23.4 \times 10^{-6} \mathrm{~cm}^{2} / \mathrm{s}\right)$ and in the control group 6.3 $\times 10^{-6}\left(3.4 \times 10^{-6}\right) \mathrm{cm}^{2} / \mathrm{s}$ (mean (SD); range $\left.3.0-12.1 \times 10^{-6} \mathrm{~cm}^{2} / \mathrm{s}\right)$.

Visual acuity of nine patients was $20 / 25$ or better including one patient with drusen $>500$ $\mu \mathrm{m}$ (Table 1). Of the four patients with the highest values of $\mathrm{P}^{\text {(pas) }}$, two had the most reduced visual acuity (20/100) whereas the other two only had slightly affected visual acuity (Table 1). In the control group visual acuity was $\geqslant 20 / 20$ in seven out of 10 subjects.

\section{Discussion}

The study investigated whether the early state of ARM was related to any changes in active or passive transport of fluorescein across the blood-retina barrier. The study showed no overall difference between patients and controls in passive permeability or unidirectional 
permeability because of outward active transport. The combination of very large drusen and pigment clumps located above drusen was associated with very high passive permeability indicating a deterioration of the blood-retina barrier. The main topics to be discussed below include the influence of drusen localisation on the fluorometric results, the role of early degenerative vitreous changes in the large variance of the unidirectional permeability, and the theory of a hydrophobic barrier at the level of Bruch's membrane in relation to the results of this study.

INFLUENCE OF DRUSEN LOCALISATION ON FLUOROMETRIC PARAMETERS

Fluorometric measurements are recorded in the optical axis and will therefore, to a higher extent, reflect the transport properties of fluorescein in the central rather than the peripheral areas of macula. ${ }^{28}$ This is regarded as a minor error. As mentioned earlier, drusen changes are assumed to involve diffuse dysfunction of the retinal pigment epithelium. Further, all patients, with the exception of two, had drusen localised to the central area within the inner circle.

UNIDIRECTIONAL PERMEABILITY IN EARLY ARM

The study demonstrated no significant difference in unidirectional permeability comparing the patient and control groups. Incipient degenerative changes with an inhomogeneous vitreous may cause an uncertainty in the determination of the diffusion coefficient and thereby in the calculation of the unidirectional permeability. Comparing the elder control group in this study with 14 young healthy subjects investigated in a previous study ${ }^{15}$ (data normally distributed), the variance of the diffusion coefficient of the elder control group was significantly higher (SD of $\ln (\mathrm{D}): 0.5341 v$ $0.1627)$. An uncertainty in the determination of the diffusion coefficient and thereby in the unidirectional permeability may have masked an intergroup difference and indicates a methodological problem. Based on this study it is therefore not possible to conclude whether early ARM is related to a decreased active transport of fluorescein caused by changes in the pigment epithelium.

PASSIVE PERMEABILITY IN EARLY ARM

The passive permeabilities across the bloodretina barrier of the patient and control group were not significantly different. However, comparing patient data with the mean and standard deviation of the control group, there were four people in the patient group with permeabilities at $3 \mathrm{SD}$ above the average. The results of the control group are assumed to be representative for healthy subjects and they were not significantly different from the results of the earlier study of 14 younger healthy participants ${ }^{15}$ (Mann-Whitney test).

The four patients differed morphologically from the remaining patients by having very large drusen $(>500 \mu \mathrm{m})$ combined with focal hyperpigmentation of varying size. One patient with smaller drusen and superjacent focal hyperpigmentation and a few patients with very large $>500 \mu \mathrm{m}$ drusen without focal hyperpigmentation did not show any apparent increase in passive permeability. No previous fluorometric studies of the early changes have been performed using the present classification system. One earlier fluorometric study did not differentiate between hard and soft drusen, and since the drusen in that study were unspecified regarding size, hyperpigmentation, etc, a direct comparison is impossible. ${ }^{29}$

The apparent abnormally high passive permeabilities in patients with very large drusen and intraretinal superjacent pigment clumps indicate a deterioration of the blood-retinal barrier. The study does not allow to assess whether a deterioration of the barrier, comprising the pigment epithelium and the endothelium of the retinal vessels, is due to changes in the pigment epithelium or to secondary changes in the retinal vessels. The histological changes are found, however, at the level of the RPE. Hyperpigmentation and large drusen are seen as risk factors for the development of neovascular $\mathrm{AMD}^{30-32}$ and it can't be ruled out that leakage from small, not yet visible, neoformation of vessels may contribute to the high permeabilities. Thus histopathological studies indicate that small neoformations of vessels are often not recognised in vivo. ${ }^{2}$

BRUCH'S MEMBRANE AS HYDROPHOBIC BARRIER It has been argued that deposits in Bruch's membrane may present a hydrophobic barrier that affects the exchange of hydrophilic matter and fluids between the choroid and the pigment epithelium and thus predisposes to pigment epithelial detachment. ${ }^{11}{ }^{11}$ In this study we found no reduced passive permeability of fluorescein across the blood-retina barrier. Presumably the passive permeability for fluorescein across Bruch's membrane, despite drusen changes, is still very high compared with the permeability across the pigment epithelium. Thus, even considerable changes in the permeability across Bruch's membrane may not lead to measurable changes in the passive permeability of the blood-retina barrier. The equivalent permeability of a combined membrane approaches the value of the membrane with the lowest permeability in case of a very large difference in the permeability of the two membranes. ${ }^{26}$

In the case of facilitated or active transport of hydrophilic substances across the pigment epithelium in the direction retina to blood, the rate of removal depends on the concentration on the apical side of the pigment epithelium and is presumably independent of the permeability across the Bruch's membrane. The deposits are therefore presumably without influence on the values of the unidirectional permeability.

This does not preclude lipid deposits in Bruch's membrane from predisposing pigment epithelium detachment by hampering the passage of ions and fluids pumped from the retina across the pigment epithelium. Further, the deposits in Bruch's membrane could affect the 
access from blood to retina of hydrophilic matter which passes the pigment epithelium by facilitated or active transport where the permeability across the Bruch's membrane could become speed limiting.

Since fluorescein is a fairly small molecule of $332 \mathrm{u}$ it is also conceivable that the drusen deposits could have a barrier role in the evolution of age-related maculopathy by restraining the access of proteins and other larger molecules.

VISUAL ACUITY

In accordance with earlier studies of patients with early age related macular degenerative changes, the majority of patients had normal or almost normal visual acuity. The lowest visual acuity of 20/100 was measured in two of the four patients with very large drusen, focal superjacent hyperpigmentations, and elevated values for passive permeability. A normal visual acuity does not preclude that the visual power may otherwise be affected. Thus, drusen changes in patients with normal visual acuity have been shown to be associated with reduced colour contrast sensitivity. ${ }^{33}$

\section{Conclusion}

The study did not demonstrate any change in the outward active transport of fluorescein across the blood-retina barrier in early ARM but may indicate elevated passive permeability in risk patients with a characteristic morphology with large drusen and pigment clumps located above drusen. The elevated passive permeability may reflect early neovascular formation of vessels that are not yet visible by fluorescein angiography. We recommend a permeability study of subgroups of ARM patients with and without very large drusen and pigment clumps and patients with known subfoveal neovascularisation. It would be interesting to include measurements of the retinal thickness which may give indications to the presence of retinal oedema and the existence of neovascularisations.

The authors thank Hans Henrik Petersen for photographic assistance. Professor Ove Sten-Knudsen is thanked for helpful comments. The study was supported by grants from the Jasha Foundation and Hotelejer Carl Larsen og hustru Nicoline Larsens Mindelegat.

1 Bird AC, Bressler NM, Bressler SB, et al. An international classification and grading system for age-related maculopathy and age-related macular degeneration. Surv Ophthalmol 1995;39:367-74

2 Green WR, Enger C. Age-related macular degeneration histopathologic studies. The 1992 Lorenz E Zimmerman Lecture. Ophthalmology 1993;100:1519-35.

3 Sarks SH. Drusen and their relationship to senile macular degeneration. Aust f Ophthalmol 1980;8:117-30.

4 Young R. Pathophysiology of age-related macular degeneration. Surv Ophthalmol 1987;31:291-306.

5 Sheraidah G, Steinmetz R, Maguire J, et al. Correlation between lipids extracted from Bruch's membrane and age. Ophthalmology 1993;100:47-51.
6 Ishibashi T, Sorgente N, Patterson R. Pathogenesis of drusen in the primate. Invest Ophthalmol Vis Sci 1986;27: 184-93.

7 Sarks JP, Sarks SH, Killingsworth MC. Evolution of soft drusen in age-related macular degeneration. Eye 1994;8: 269-83.

8 Green WR, McDonnell PJ, Yeo JH. Pathologic features of senile macular degeneration. Ophthalmology 1985;92:61527.

9 Pauleikhoff A, Harper CA, Marshall J, et al. Aging changes in Bruch's membrane. Ophthalmology 1990;97:171-8.

10 Bird AC, Marshall J. Retinal pigment epithelial detachment in the elderly. Trans Ophthalmol Soc UK 1986;105:674-82.

11 Starita C, Hussain AA, Pagliarini S, et al. Hydrodynamics of ageing Bruch's membrane: implications for macular disease. Exp Eye Res 1996;62:565-72.

12 Engler CB, Sander B, Larsen M, et al. Fluorescein transport across the human blood-retina barrier in the direction vitreous to blood. Quantitative assessment in vivo. Acta Ophthalmol 1994;72:655-62.

13 Cunha-Vaz J. The blood-ocular barriers. Surv Ophthalmol 1979;23:279-96.

14 Moldow B, Sander B, Engler C, et al. The effect of acetazolamide on leakage and outward active transport of fluorescein across the blood-retinal barrier in retinitis pigmentosa complicated by macular oedema. Graefes Arch Clin Exp Ophthalmol. 1998;236:881-9.

15 Moldow B, Sander B, Larsen M, et al. Effects of acetazolamide on passive and active transport of fluorescein across
the normal BRB. Invest Ophthalmol Vis Sci 1999;40:17705.

16 Dalgaard P, Larsen M. Fitting numerical solutions of differential equations to experimental data: a case study and some general remarks. Biometrics 1990;46:1097-9.

17 Larsen M, Dalgaard P, Lund-Andersen H. Differential spectrofluorometry in the human vitreous: blood-retina barrier permeability to fluorescein and fluorescein glucuronide. Graefes Arch Clin Exp Ophthalmol 1991;229:350-7.

18 Lund-Andersen $\mathrm{H}$, Krogsaa B, la Cour M, et al. Quantitative vitreous fluorophotometry applying a mathematical model of the eye. Invest Ophthalmol Vis Sci 1985;26:698710 .

19 Cunha-VaZ JG, Maurice DM. The active transport of fluorescin by the retinal vessels and the retina. F Physiol 1967; 191:467-86

20 Stone RA, Wilson CM. Fluorescein transport in the anterior uvea. Invest Ophthalmol Vis Sci 1982;22:303-9.

21 Fatt I, Shantinath K. Flow conductivity of retina and its role in retinal adhesion. Exp Eye Res 1971;12:218-26.

22 Araie M, Maurice DM. The loss of fluorescein, fluorescein glucuronide and fluorescein isothiocyanate dextran from the vitreous by the anterior and retinal pathways. Exp Eye Res 1991;52:27-39.

23 Blair N P, Zeimer RC, Rusin MM, et al. Outward transport of fluorescein from the vitreous in normal human subjects. Arch Ophthalmol 1983;101:1117-21.

24 Zeimer RC, Noth JM. A new method of measuring in vivo the lens transmittance, and study of lens scater, fluorescence and transmittance. Ophthalmic Res 1984;16:246-55.

25 Zeimer RC, Blair NP, Cunha-VaZ JG. Pharmacokinetic interpretation of vitreous fluorophotometry. Invest Ophthalmol Vis Science 1983;24:1374-81.

26 Sten-Knudsen O. In: Stoftransport, membranpotentialer og elektriske impulser over biologiske membraner. Arhus: Akademisk Forlag, 1995: Chapter 2.34 and 2.732.

27 Armitage P, Berry G. Statistical methods in medical research. 3rd ed. Oxford: Blackwell Scientific, 1994:197-8 and 397-8.

28 Dalgaard P, Barker VA, Lund-Andersen H. Vitreous fluorophotometry: mathematical analysis of the efect of peripheral leakage on axial scans. Invest Ophthalmol Vis Sci 1989;30:1522-6.

29 Merin S, Blair N, Tso MO. Vitreous fluorophotometry in patients with senile macular degeneration. Invest Ophthalmol Vis Sci 1987;28:756-9.

30 Bressler SB, Maguire MG, Bressler NM, et al. Relationship of drusen and abnormalities of the retinal pigment epithelium to the prognosis of neovascular macular degeneration. Arch Ophthalmol 1990;108:1442-7.

31 Smiddy WE, Fine SL. Prognosis of patients with bilateral macular drusen. Ophthalmology 1984;91:271-7.

32 Strahlman ER, Fine SL, Hillis A.The second eye of patients with senile macular degeneration. Arch Ophthalmol 1983; 101:1191-3.

33 Frennesson C, Nilsson UL, Nilsson SE. Color contrast sensitivity in patients with soft drusen, an early stage of ARM. Doc Ophthalmol 1995;90:377-86. 